\title{
Infant walker use, injuries, and motor development
}

\author{
M M Thein, J Lee, V Tay, S L Ling
}

\begin{abstract}
Objectives-To study the impact of infant walker use on motor development and injuries.

Population-One hundred and eighty five parents or primary care givers who attended a Singapore government polyclinic from September 1993 to February 1994, with their infants between 7 to 10 months, for a developmental assessment session.
\end{abstract}

Setting-A government polyclinic in Singapore.

Methods-The parent or primary care giver answered questions pertaining to infant walker use and injuries attributed to its use. Each infant was then given the Singapore modified version of the Denver Developmental Screening Test (DDST-S), along with a full clinical examination; both testers were blinded to walker use.

Results-One hundred and sixty seven $(90 \%)$ of 185 infants used walkers regularly, and $21(12.5 \%)$ of the users had one or more injuries. Most injuries were minor, such as bruises and swellings on the head, forehead, face, and cheeks. None of the children who did not use walkers showed any abnormal DDST-S results whereas $18(10.8 \%)$ of the 167 walker users had either abnormal or questionable DDST-S results.

Conclusions-12.5\% of walker users had one or more injuries and walker use may also delay the child's motor development. These findings will help the physician or nurse in primary care settings to advise parents about the potential hazards of walker use.

(Injury Prevention 1997; 3: 63-66)

Keywords: child development; infant walker use; health education.

Although baby walkers have been in use for many years, they only became widespread after the post world war II baby boom. A typical walker consists of a seat suspended between a wooden or metal frame on wheels. The infant sitting in the walker can touch the floor with the feet and can thereby move around in various directions before knowing how to walk. Infant walkers are used by many parents to keep children occupied and happy.
There was early recognition of potential hazards associated with the use of these walkers. ${ }^{1-4}$

The continuing use of walkers appears to be motivated by the notion that not only does the walker keep the child safely occupied, but that it also facilitates the infant's ability to walk. ${ }^{1}$ Available evidence, however, does not support this view. Indeed, a large number of minor and some major injuries, such as head trauma, have been attributed to walker use. ${ }^{2}$ Nor does the use of walkers appear to promote early ambulation. A study of six pairs of twins by Kauffmann and Ridenour found that infants who never use the walker started walking earlier than their siblings. ${ }^{5}$ There are no studies to date that address the potentially adverse impact of walker use on the development of apparently normal infants or those who are at high risk for neurological delay in ambulation.

This study was carried out to ascertain the prevalence of infant walker use in Singapore and to explore their possible effects on injuries and on motor development.

\section{Subjects and methods}

One hundred and eighty five parents or primary care givers who attended a government polyclinic developmental assessment session with their infants aged 7 to 10 months from September 1993 to February 1994 were studied. They were given a short questionnaire by a trained interviewer regarding walker usage, average number of hours used per day, and injuries sustained. Each infant was then administered a developmental screening test either by a fully trained nurse or by one of the authors (MMT) who also carried out clinical examination of all the infants. Administrators of the test were blinded to the results of the injury-walker use questions. The test was the Singapore version of the revised Denver Developmental Screening Test, ${ }^{6}$ henceforth referred to as DDST-S.

The DDST-S, which seeks to assess development in gross motor, fine motor, speech, language, personal and social sectors, has been in use for preschool age children in Singapore polyclinics since 1992. Test scores are categorised as 'normal' (either no 'delay' or 'caution' in development in all sectors, or only a 'caution' in the entire test), 'questionable' (one delay and one or more cautions), or 'abnormal' (two or more 'delays'). Further 
details of the meaning of DDST-S scores and the terms 'delay' and 'caution' are discussed elsewhere. ${ }^{6}$

The primary goal of the statistical analysis was to assess duration (average hours per day) of walker use (exposure variable) on injury and delay in development (response variables). Initially we used the $\chi^{2}$ trend test for monotonic relationship between exposure and response. ${ }^{7}$ These univariate analyses, however, do not take into account potential confounding factors - ethnicity, sex, and age - which may distort the apparent exposure-response relationship. It is, therefore, crucial to evaluate the relationship after adjusting for possible confounding. Cox's proportional hazards model adopted for cross sectional data was used for this task. ${ }^{8-10}$

\section{Results}

Of the 185 infants included in the study sample, 167 (90\%) used walkers regularly. There are 14 polyclinics in Singapore with services for developmental assessment; they cater for $60 \%$ of the preschool age group. The majority of children are from middle and lower income families. Our study was conducted at one such polyclinic, which is representative of all the others in Singapore. The percentage of walker users according to sociodemographic attributes is summarised in table 1 . The data show that $90 \%$ of infants in Singapore, irrespective of socioeconomic status, use walkers.

WALKER USE AND INJURIES

Of the 167 infants who used walkers, 21 $(12.5 \%)$ had one or more injuries. All the

Table 1 Walker users according to personal and sociodemographic attributes

\begin{tabular}{lr}
\hline Attribute & No (\%) \\
\hline Ethnic group & \\
Chinese (92) & $87(95)$ \\
Malay (85) & $73(86)$ \\
Indian (6) & $5(83)$ \\
Mother's occupation & $85(91)$ \\
$\quad$ Working mother (93) & $82(89)$ \\
$\quad$ Housewife (92) & $56(95)$ \\
Mother's education & $111(88)$ \\
$\quad$ Primary (59) & $48(92)$ \\
Secondary or higher (126) & $117(89)$ \\
Father's education & $101(92)$ \\
$\quad$ Primary (52) & $65(88)$ \\
Secondary or higher (131) & $87(94)$ \\
Child's age (months) & $80(87)$ \\
7-8 (110) & \\
9-10 (74) & \\
Child's sex & Male (93) \\
Female (92) & \\
\hline
\end{tabular}

Table 2 Walker use, injuries, and DDST-S results; values are number (\%)

\begin{tabular}{llll}
\hline & \multicolumn{3}{l}{ Hours/day of walker use } \\
\cline { 2 - 4 } & $<1$ & $1-2$ & $>2$ \\
& $(n=29)$ & $(n=50)$ & $(n=88)$ \\
\hline Injuries & $3(10.3)$ & $5(10.0)$ & $13(14.8)$ \\
Abnormal or questionable DDST-S & $2(6.8)$ & $8(16.0)$ & $8(9.1)$ \\
\hline
\end{tabular}

injuries reported were minor, such as bruises or bumps. The per cent of injuries according to hours of walker use is shown in table 2 . Although infants who used the walker longer than two hours per day reported a higher pep cent of injuries $(14.8 \%)$ than those who use the walker for shorter periods $(10.3 \%$ and $10.0 \%$ ), the gradient was not statisticall significant $(\mathrm{p}=0.43)$.

WALKER USAGE AND MOTOR DEVELOPMENT $\stackrel{\mathbb{\perp}}{\Omega}$ Whereas all 18 infants who did not use a walk showed normal DDST-S developmental r $\xi$ sults, $12(7.2 \%)$ of the 167 who used walkers showed abnormal, and six (3.6\%) showerd questionable, DDST-S results. Of the lattert 18 infants, 17 showed a delay in gross motén development and one showed delay in speecti and language development. The per cent of infants with abnormal or questionable DDST $S$ test results according to hours of walket usage is also shown in table 2 and no gradie is apparent.

\section{ADJUSTMENT FOR CONFOUNDING}

The prevalence ratios of hours per day walker use on injury, abnormal DDST-S results and gross motor delay, adjusted for ethnicity, sex, and age are presented in table The prevalence ratios and their $95 \%$ conf dence intervals were estimated using Cox proportional hazards regression model adoptea for cross sectional data. ${ }^{8-10}$

An infant who used the walker for more than two hours per day, on average, is 1.43 times more likely to have incurred an injury than one who used the walker for less than one hour per day. Adjustment for confounding did n\& materially alter the result. Also, an infant who used a walker for longer than one hour per das on average, showeđ a discernibly elevated risk of having an abnormal or questionable DDST $S$ score and a gross motor delay compared with those who used the walker for less than one hour per day. This result is more pronounce after adjustment for confounding, that is the adjusted prevalence ratios are greater than the observed prevalence ratios (table 3 ).

Table 3 Prevalence ratio (PR) of injury, abnormal or 0 questionable DDST $-S$ result, and gross motor delay in relation to hours/day of walker usage: results of Cox regression

\begin{tabular}{llll} 
Walker use & Observed & Adjusted & $\stackrel{0}{+}$ \\
\cline { 3 - 4 } (hours/day) & PR $(95 \%$ CI $)$ & PR $(95 \%$ CI $)$ & $\stackrel{+}{+}$ \\
\hline
\end{tabular}

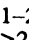

$1-2$
$>2$

$1-2$

$>2$

${ }^{\star}$ Adjusted for confounding due to ethnicity, sex, and age of the child. All the prevalence ratios are relative to less than one hour of walker use per day (the reference group).

$\mathrm{CI}=$ confidence interval. 


\section{Discussion}

With the eradication and control of many infectious diseases and undernutrition, physicians looking after children spend more time counselling parents with regard to growth, development, and injury prevention. In Singapore, injuries due to falls, burns, poisoning, drowning, and road traffic accidents have received much attention, ${ }^{11-15}$ whereas hazards attributable to walker use have been ignored. To date there are no data in Singapore on injuries related to walker use.

Our data show that infant walkers are widely used $(90 \%)$ irrespective of race, sex of the child, or socioeconomic status. In contrast, data from two US studies show a lower prevalence of walker use (70 to $86 \%$ ). ${ }^{14}$ Our study shows that $12.5 \%$ of the walker users had one or more injuries, which is considerably lower than the 30 to $40 \%$ reported in the US studies. However the injuries in our study were minor, needing no treatment or, at most, only self treatment. This is in contrast to one US study that reported both minor and serious injuries. $^{3}$

In our study, the possibility of underreporting on the frequency as well as severity of injuries attributable to walker use cannot be excluded. The parents and care givers may feel embarassed to admit such injuries. Conversely as the reported injuries were mainly minor, this may be due to close supervision by the parents and care givers or the absence of stairs in the homes of many families in the study. Another reason for the mild injuries is that the infants studied came to a well child clinic. Had the study been carried out at an accident and emergency department, it is most likely that more serious injuries would have been reported.

Our data also showed that walker use may have affected the child's development. Eighteen $(10.8 \%)$ of the 167 walker users exhibited abnormal or questionable DDST-S results, and $17(95 \%)$ of these showed delay in the gross motor sector, such as being unable to sit without support, bear weight, or stand while holding on. The clinical examination was normal in these infants, indicating they did not have muscular bone, joint, endocrine, or neurological problems. The parents of these infants were advised not to put them in walkers, and when they returned for a follow up visit two months later, all had reverted to normal development. The delay in development may or may not be due to the effect of walker alone; it may also be due to familial delay.

Previous studies have shown that walker use not only does not lead to earlier walking, as is commonly believed, but may even delay the onset of walking. ${ }^{516}$ As the infants in our study were between 7 and 10 months, they were not of walking age. Their delay was mainly in sitting. Of the 18 who did not use walkers, all showed normal DDST-S results. The two reasons given for not using walkers were that some parents had experienced injuries with their older children or that they had attended parent education talks that highlighted the dangers of walkers.
Studies from other countries have also shown that most walker injuries are minor, but there were also some serious injuries reported such as head injuries, ${ }^{17}$ burns, or scalds. ${ }^{18} 19$ This is due to another problem associated with the use of these walkers. Walkers give the infants an advanced degree of mobility before the normal developmental stage of walking. The usual age of babies that are put in walkers is from 5 months to 10 months. With the walker the baby can move from one corner of the room to the other, or from one room to another very quickly. In addition, some parents or care givers use walkers as a substitute for supervision. The figures from Britain and the US have shown that there is an increase in cases of children with burns and scalds related to walkers. ${ }^{17}$ The babies in walkers reversed into gas fires; went to the kitchen and hit the oven, and one even pulled the kettle down. Recently, in Singapore we also had a similar case. A 6 month old baby was put in the walker in the living room. The mother was away for only a few minutes in the other room. The baby in the walker wheeled himself into the kitchen, pulled the porridge pot, which was on the fire, and was scalded. The baby was rushed immediately to hospital but died a few days later.

\section{Implications for prevention}

As pointed by Greensher and Mofenson, ${ }^{20}$ by increasing the child's mobility there is more opportunity to get into trouble. As there is no benefit associated with their use, and our data reiterate their dangers, health education should be given to parents and physicians need to warn parents about these hazards. They may also stress the possible effect of walkers on the child's motor development. Health care professionals should advise parents to use a crib or playpen rather than a walker when it is necessary for the infant to be out of their sight for a moment. ${ }^{21}$

We would like to thank the following nurses for their generous assistance throughout the study; Ms Norhana Binti Abdul Rahman, Ms Teng Mee Ying, Ms Chin Soo Ngoh and Ms Heng Siew Tiang of Bedok Polyclinic, and Mrs Julie Chew of the Department of Community, Occupational and Family Medicine, National University of Singapore.

1 Fazen LE, Felizberto PI. Baby walker injuries. Pediatrics 1982; 70: 106-9.

2 Kavaragh CA, Banco L. The infant walker; a previously unrecognized health hazard. Am $\mathcal{F}$ Dis Child 1982; 136: 205-6.

3 Rieder MJ, Schwartz C, Newman J. Patterns of walker use and walker injury. Pediatrics 1989; 78: 488-93.

4 American Medical Association Board of Trustees. Use of infant walkers. Am $\mathcal{F}$ Dis Child 1991; 145: 933-4.

5 Kauffman IB, Ridenour M. Influence of infant walkers on onset and quality of walking pattern and locomotion: an onset and quality of walking pattern and locomotion: an
electromyograhpic investigation. Percept Mot Skills 1977; 45: $1323-9$.

$6 \mathrm{Lim}$ HC, Chan T, Yoong T. Standardization and adaption of the Denver Development Screening Test (DDST) and Denver II for use in Singapore. Singapore Med f 1994; 35 $156-60$.

7 Lee J. Comparing several proportions when the exposure variable is either ordinal or ratio which had been grouped into discrete classes. Comput Biol Med 1994; 24: 211-20.

8 Lee J. Odds ratio or relevative risk for cross-sectional data? Int $\mathcal{f}$ Epidemiol 1994; 23: 210-3.

9 Lee J, Chia KS. Estimation of prevalence rate ratios for cross-sectional data: an example in occupational epidemiology. Br $\mathcal{F}$ Indust Med 1993; 50: 861 -2. 
10 Axelson $\mathrm{O}$, Fredriksson M, Ekberg K. Use of the prevalence ratio $\mathrm{v}$ prevalence odds ratio as a measure of risk in cross sectional studies. Occup Environ Med 1994; 51: 574.

11 Emmanuel S. Epidemiology of injuries in Singapore. Ann Acad Med Singapore 1991; 20: 190-5.

12 Lee ST. Two decades of specialised burns care in Singapore, 1961 - 1982. Ann Acad Med Singapore 1982; 11: 358-65.

13 Ngim RCK. Burns in children. Ann Acad Med Singapore 1992; 21: $665-71$

$14 \mathrm{Ng} \mathrm{SC}$, Chao TC, How J. Death by accidental drowning in Singapore, 1973 - 1976. Singapore Med f 1978; 19: 14-9.

15 Thein MM, Lee J. Road safety education for school children. World Health Forum 1993; 14: 407-9.
16 Holm WA, Harthun-Smith L, Tada WL. Infant walkers and cerebral palsy. Am $\mathcal{F}$ Dis Child 1983; 137: $189-90$.

7 Stoffman JM, Bass MJ, Fox AM. Head injuries related to the use of baby walkers. Can Med Assoc f 1984; 131: 573-5. 18 Birchall MA, Henderson HP. Baby walkers and infant burns. $B M F$ 1988; 296: 1641 .

19 Millar R, Colville J, Hughes NC. Burns to infants usinf walker aids. Injury 1975; 7: 8-10.

20 Greensher J, Mofenson HC. Injuries at play. Pediatr Ci North Am 1985; 32: 1: $127-39$.

21 Twinkoff A, Parks PL. Prevention strategies for infa walker related injuries. Public Health Rep 1993; 6: 784-

\section{Editorial Board Member: brief biography}

YVONNE ROBITAILLE

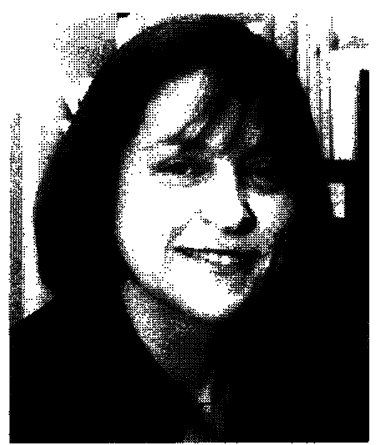

Yvonne Robitaille is an epidemiologist at the Public Health Department of the Montréal-Centre region in Québec, Canada. She is a faculty member at both the Department of Epidemiology and Biostatistics of McGill University and Department of Social Medicine of the University de Montréal. She graduated from McGill University in 1983 with a PhD in epidemiology, and completed a postdoctoral fellowship in injury epidemiology at The Johns Hopkins University in 1986.

Realising that relatively little was known about the rate of injuries in the province of Québec, and aware that existing Québec data had yet to be explored, she became involved in several surveillance studies. One aimed at assessing the scope of the problem in terms of mortality, hospitalization, and disability. A second focused on describing regional differences. Another concentrated on assessing the quality of data on injury hospitalisation. A more recent study focused on making better use of the data gathered in paediatric hospital emergency rooms. On two occasions she has province-wide population survey data to describe the experience of injuries in all age groups.

Her research has also addressed the risk factors for specific types of injuries including children's home and playground injuries and falls in the elderly. Importantly, her work also includes the evaluation of some preventive interventions at the community level. One of these involved an infant car seat loan program, another comprised strategies to prevent falls among the elderly people.

Dr Robitaille is currently a member of the scientific committee of Santé Québec (the province's official health survey body) and of the Reseau francophone de prevention des traumatises et des accidents, and is involved in the activities of the WHO Collaborating Centre for safety promotion and injury prevention. She has published widely in both the English and French scientific literature on injury prevention. 